\title{
Hospital Length of Stay and All-Cause 30-Day Readmissions Among High-Risk Medicaid Beneficiaries
}

\author{
Ishveen Chopra, MS*, Tricia Lee Wilkins, PharmD, MS, PhD, Usha Sambamoorthi, PhD
}

West Virginia University, School of Pharmacy, Morgantown, West Virginia.

This study examined the association between index hospitalization characteristics and the risk of all-cause 30-day readmission among high-risk Medicaid beneficiaries using multilevel analyses. A retrospective cohort with a baseline and a follow-up period was used. The study population consisted of Medicaid beneficiaries (21-64 years old) with selected chronic conditions, continuous fee-for-service enrollment through the observation period, and at least 1 inpatient encounter during the follow-up period $(\mathrm{N}=$ $15,806)$. The outcome of 30 -day readmission was measured using inpatient admissions within 30-days from the discharge date of the first observed hospitalization. Key independent variables included length of stay, reason for admission, and month of index hospitalization (seasonality). Multilevel logistic regression that accounted for beneficia- ries nested within counties was used to examine this association, after controlling for patient-level and county-level characteristics. In this study population, $16.7 \%$ had allcause 30-day readmissions. Adults with greater lengths of stay during the index hospitalization were more likely to have 30-day readmissions (adjusted odds ratio [AOR]: 1.03, $95 \%$ confidence interval [Cl]: 1.02-1.04). Adults who were hospitalized for cardiovascular conditions (AOR: 1.20, 95\% $\mathrm{Cl}$ : 1.08-1.33), diabetes (AOR: 1.23, 95\% Cl: 1.10-1.39), cancer (AOR: 1.55, 95\% Cl: 1.26-1.90), and mental health conditions (AOR: 2.17, 95\% Cl: 1.98-2.38) were more likely to have 30 -day readmissions compared to those without these conditions. Journal of Hospital Medicine 2016;11:283-288. ( 2015 Society of Hospital Medicine
Hospital readmissions that occur within 30 days of discharge are an important measure for assessing performance of the healthcare system and the quality of patient care. ${ }^{1,2}$ According to the Healthcare Cost and Utilization Project (HCUP), there were approximately 3.3 million adults with all-cause 30-day readmissions in the United States in 2011, incurring nearly $\$ 41.3$ billion in hospital costs. ${ }^{3}$ Reducing 30-day readmissions has become a priority for payers, providers, and policymakers seeking to achieve improved quality of care at lower costs.

The implementation of the Affordable Care Act (ACA) provided the Centers for Medicare \& Medicaid Services (CMS) statutory authority under the Hospital Readmissions Reduction Program to reduce payments for certain hospital readmissions that it deemed avoidable. $^{4}$ Although initial focus was on Medicare readmissions related to heart failure, myocardial infarction, and pneumonia, CMS is now considering expanding the list beyond the 3 conditions covered by the program. ${ }^{4,5}$ Therefore, it is important to understand major risk factors for readmissions in beneficiaries with chronic conditions.

\footnotetext{
*Address for correspondence and reprint requests: Ishveen Chopra MS, West Virginia University, School of Pharmacy, Morgantown, WV 26506; Telephone: 412-427-5627; Fax: 304-293-2529;

E-mail: ishveenkc@gmail.com

Additional Supporting Information may be found in the online version of this article.

Received: June 26, 2015; Revised: November 2, 2015; Accepted: November 15, 2015

2015 Society of Hospital Medicine DOI 10.1002/jhm.2526

Published online in Wiley Online Library (Wileyonlinelibrary.com).
}

Medicaid consists of the largest number of beneficiaries among all payers in the United States, with approximately 62 million beneficiaries in 2013.5 The Medicaid population is further expected to increase with the coverage expansions under the ACA. In addition, the state Medicaid programs incur an estimated $\$ 374$ billion in healthcare expenditures and provide healthcare services to the vulnerable, indigent, and disabled. It is estimated that $61 \%$ of adult Medicaid beneficiaries have chronic or disabling conditions that place them at an increased risk of hospitalization. ${ }^{6} \mathrm{~A}$ series of HCUP statistical briefs reported several findings. First, Medicaid all-cause readmission rates were comparable with Medicare but double the rate of private insurance. ${ }^{7}$ Second, for readmissions following nonsurgical hospitalizations, 30-day Medicaid readmission rates were higher than Medicare and private insurance for both acute and chronic conditions. ${ }^{1}$ The effects of such costly utilization patterns, for this large and growing population necessitates heightened attention under healthcare reform.

The balance between hospital efficiency and quality of care is another crucial aspect for our healthcare system. However, length of stay (LOS), a proxy marker for efficiency, may conflict with hospital readmission rates, an indicator of quality. Further, CMS plans to bundle 30-day readmission rates to reimbursement for the index hospitalization. ${ }^{8}$

The effect of LOS on readmission rates is complex, and previous studies have provided conflicting data regarding the relationship between LOS and subsequent readmission risk. Some indicate that shorter LOS is associated with a higher risk of readmission, ${ }^{8,9}$ 
whereas others suggest that extended LOS is associated with a higher risk of readmission. ${ }^{10-12}$ However, most research on readmissions has focused on Medicare beneficiaries. ${ }^{11,13,14}$ The readmission patterns of Medicaid beneficiaries differ from those of the geriatric Medicare beneficiaries, from a clinical and socioeconomic perspective. Considering the importance of 30-day readmission for payers and policy makers, there is a need to understand the role of LOS and implications for treatment and management strategies.

Our study examined the association between index hospitalization characteristics (LOS and reason for admission) and all-cause 30-day readmission risk in fee-for-service high-risk Medicaid beneficiaries. The study is limited to patients with selected chronic conditions and examines the differentiating factors within this high-risk population. For the purpose of our study, variables were selected based on a priori knowledge and Andersen's behavioral model of health service utilization. This model suggests that potential health service use is determined by interactions among predisposing (demographics, index hospitalization characteristics), enabling (county level [eg, socioeconomic status]), and need (health status) characteristics of individuals and also the healthcare systems in the communities where they reside. ${ }^{15}$

\section{METHODS}

\section{Study Design}

A retrospective cohort approach was used with baseline and follow-up periods. The baseline period was defined as the admission date of the index hospitalization (first observed hospitalization) between January 1, 2007 and December 31, 2007. Patients were followed for 180 days after discharge date of the associated index hospitalization.

\section{Data Source}

Medicaid administrative claims files from California, Illinois, New York, and Texas, between 2006 and 2008, were used. The personal summary file included information on demographics, Medicaid enrollment, and eligibility status. Outpatient and Inpatient files included claims for services provided in ambulatory and inpatient settings and contained International Classification of Diseases, 9th Revision, Clinical Modification codes. Information on county-level characteristics were obtained from the 2009 Area Health Resource File (AHRF), which was linked to Medicaid administrative claims files using state and county codes where each beneficiary resided.

\section{Study Population}

The study population consisted of nonelderly (21-64 years old) fee-for-service Medicaid-only beneficiaries with selected chronic conditions and continuous enrollment during baseline and follow-up period (Figure 1). Analyses were restricted to those who had

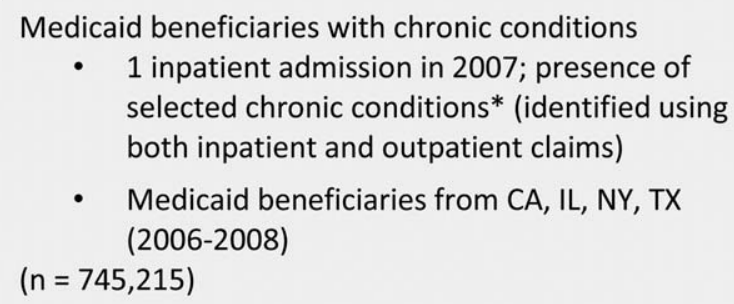
selected chronic conditions* (identified using both inpatient and outpatient claims)

- Medicaid beneficiaries from CA, IL, NY, TX (2006-2008)

$(n=745,215)$

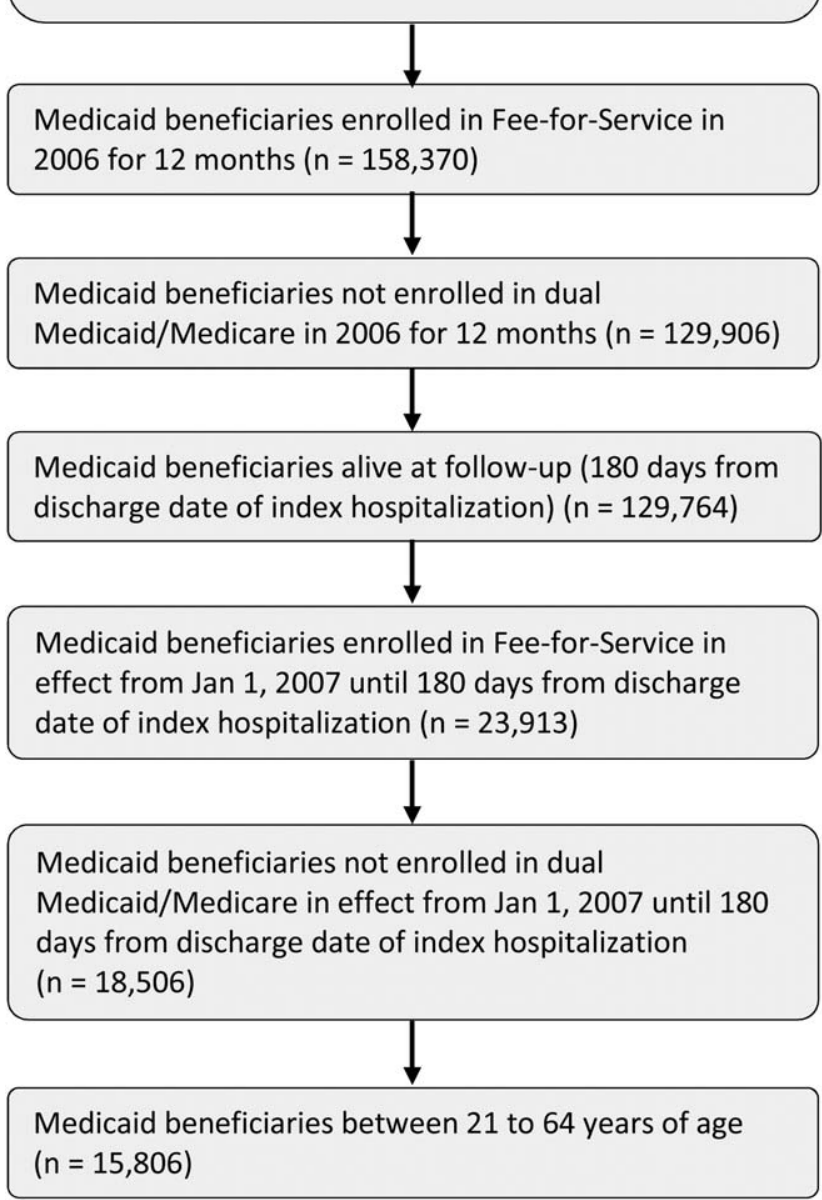

FIG. 1. Schematic presentation of selection criteria. *Selected chronic conditions: asthma, arthritis, cardiac arrhythmias, coronary artery disease, cancer, congestive heart failure, chronic kidney disease, chronic obstructive pulmonary disease, dementia, diabetes, hypertension, hyperlipidemia, hepatitis, human immunodeficiency virus osteoporosis, stroke, depression, schizophrenia, and substance use disorders.

at least 1 inpatient admission in 2007 and were conducted at the person-level.

For the purpose of this study, Medicaid beneficiaries with 19 chronic conditions were selected: asthma, arthritis, cardiac arrhythmias, coronary artery disease, cancer, congestive heart failure, chronic kidney disease, chronic obstructive pulmonary disease, dementia, diabetes, hypertension, hyperlipidemia, hepatitis, human immunodeficiency virus osteoporosis, stroke, depression, schizophrenia, and substance use disorders. These conditions were identified based on the strategic framework developed and adopted by the Department of Health and Human Services for research, policy, program, and practice. ${ }^{16}$ 


\section{Dependent Variable}

Individuals were categorized into 2 groups, those with and without all-cause 30-day readmission. All-cause 30-day readmission was identified as subsequent hospitalization within 30 days of discharge date of the index hospitalization.

\section{Key Independent Variables}

These were index hospitalization characteristics, where LOS was the primary independent variable, reason for admission was the secondary independent variable, and month of index hospitalization (included to control for potential seasonal effect).

\section{Other Independent Variables}

Patient-level characteristics included demographics (age, gender, and race/ethnicity) and Medicaid eligibility status (cash and medical need). Primary care access included continuity of care measured using a previously published continuity index (Modified Modified Continuity Index) and coordination of care, measured as primary care visit within 14 days of discharge date. Healthcare utilization was measured as an emergency room visit within 6 months prior to the index hospitalization.

Variables accounting for county socioeconomic status included educational attainment, per capita income, employment rate, poverty level, and metropolitan statistical area. Variables related to availability of providers and healthcare facilities were AHRF designations for primary/mental healthcare shortage areas, presence of federally qualified health centers, rural health centers, and community mental health centers. Hospital and primary care provider density was defined as total number of hospitals or primary care providers per 100,000 individuals, respectively.

\section{Statistical Techniques}

$\chi^{2}$ tests of independence were used for categorical variables and $t$ tests for continuous variables to determine group differences in patient-level and county-level characteristics and all-cause 30-day readmission. Multilevel logistic regression models, which accounted for beneficiaries nested within counties, were used to examine the association between all-cause 30-day readmission and index hospitalization characteristics. The reference group for the dependent variable was no 30-day readmission. Model 1 controlled for only patient-level characteristics. Model 2 controlled for both patient-level and county-level characteristics. In both models, county was specified as a random intercept using the GLIMMIX procedure. All analyses were conducted using SAS version 9.3 (SAS Inc., Cary, NC).

\section{RESULTS}

After the exclusion criteria, there were 15,806 Medicaid beneficiaries with selected chronic conditions and at least 1 inpatient encounter in 2007. Overall, $16.7 \%$ experienced all-cause 30-day readmissions. A description of the study population and unadjusted associations between independent variables and all-cause 30day readmission are presented in Table 1 .

Multilevel logistic regressions of all-cause 30-day readmissions are summarized in Table 2. Beneficiaries with longer LOS had significantly higher odds of 30day readmission. In addition, presence of cancer, cardiovascular conditions, diabetes, and mental health conditions at index hospitalization significantly increased the odds of readmission. In addition, beneficiaries with cash or medical need eligibility had significantly higher odds of 30-day readmission.

\section{DISCUSSION}

To the best of our knowledge, this is the first study examining patient-level and county-level characteristics associated with all-cause 30 -day readmission in Medicaid beneficiaries with chronic conditions. In addition, our findings add to the nascent literature on readmissions among Medicaid beneficiaries, with findings discussed below.

LOS has been reported as a risk factor for readmission both in elderly and nonelderly populations. ${ }^{11}$ Our findings indicate that longer LOS is associated with increased odds of 30-day readmission, which could be attributed to severity of illness at index hospitalization. ${ }^{10}$ This finding could be related to unmeasured clinical severity (our models account for some comorbidities) and socioeconomic issues (as noted in the introduction). This may have implications for discharge planning efforts and focusing on chronic disease management, which has previously shown to be effective in reducing readmissions. ${ }^{17}$ Our findings suggest 30-day readmissions can be predicted using variables that are readily available, few in number, and simple to incorporate in discharge planning. Comprehensive discharge planning which takes into account chronic conditions and index hospitalization characteristics may help organize postdischarge services, including coordination of care with physicians, medication reconciliation, follow-up care, and appropriate self-management for chronic conditions.

Our findings of increased risk of 30-day hospital readmissions as well as longer LOS among Medicaid beneficiaries with cancer, cardiovascular conditions, diabetes, and mental health conditions at index hospitalization suggests that patient complexity/poor health status increases the risk of readmission. A more focused approach in treatment of these diseases can help reduce readmissions. Integrated care management interventions after hospital discharge have been shown to reduce readmissions among those with heart disease; a coordinated care team including cardiologists, specialized nurses, and primary care physicians, and provision of integrated care following hospitalizations have shown benefit. ${ }^{18,19}$ Emerging models of delivery 
TABLE 1. Description of Study Population by All-Cause 30-Day Readmission Multistate Medicaid Fee-for-Service Beneficiaries With Selected Chronic Conditions, 2006-2008

\begin{tabular}{|c|c|c|c|}
\hline Variables & $\begin{array}{c}\text { 30-Day Readmission, } \\
2,633(16.7 \%)\end{array}$ & $\begin{array}{c}\text { No 30-Day Readmission, } \\
13,173(83.3 \%)\end{array}$ & Significance \\
\hline \multicolumn{4}{|c|}{ Demographic and Medicaid eligibility characteristics } \\
\hline Gender, N $(\%)$ & & & * \\
\hline Female & $1,715(65.1 \%)$ & $9,274(70.4 \%)$ & \\
\hline Male & $918(34.9 \%)$ & $3,899(29.6 \%)$ & \\
\hline Age group, $N(\%)$ & & & * \\
\hline 21-24 years & $301(11.4 \%)$ & $1,675(12.7 \%)$ & \\
\hline $25-34$ years & $567(21.5 \%)$ & $3,578(27.2 \%)$ & \\
\hline $35-44$ years & $517(19.6 \%)$ & $2,498(19.0 \%)$ & \\
\hline $45-54$ years & $673(25.6 \%)$ & $2,971(22.6 \%)$ & \\
\hline 55-64 years & $575(21.8 \%)$ & $2,451(18.6 \%)$ & \\
\hline Race/ethnicity, N (\%) & & & * \\
\hline Caucasian & $847(32.2 \%)$ & $3,831(29.1 \%)$ & \\
\hline African American & $988(37.5 \%)$ & $4,270(32.4 \%)$ & \\
\hline Hispanic & $608(23.1 \%)$ & $4,245(32.2 \%)$ & \\
\hline Asian/Al/PI & $39(1.5 \%)$ & $169(1.3 \%)$ & \\
\hline Other & $151(5.7 \%)$ & $658(5.0 \%)$ & \\
\hline Cash eligibility, N (\%) & $1,529(58.1 \%)$ & $6,666(50.6 \%)$ & * \\
\hline Medical need eligibility, N (\%) & $876(33.3 \%)$ & $3769(28.6 \%)$ & * \\
\hline \multicolumn{4}{|l|}{ Index hospitalization characteristics } \\
\hline Length of stay, mean [SD] & $6.62[9.09]$ & $4.29[6.35]$ & * \\
\hline \multicolumn{4}{|l|}{ Chronic conditions at admission, N (\%) } \\
\hline Arthritis/osteoporosis & $99(3.8 \%)$ & $464(3.5 \%)$ & \\
\hline Cancer & $134(5.1 \%)$ & $429(3.3 \%)$ & * \\
\hline Cardiovascular conditions & $995(37.8 \%)$ & $3,733(28.3 \%)$ & * \\
\hline COPD/asthma & $541(20.5 \%)$ & $2,197(16.7 \%)$ & * \\
\hline Diabetes & $575(21.8 \%)$ & $2,103(16.0 \%)$ & * \\
\hline HIV/hepatitis & $305(11.6 \%)$ & $1,185(9.0 \%)$ & * \\
\hline Mental health conditions & $1,491(56.6 \%)$ & $4,352(33.0 \%)$ & * \\
\hline Season of readmission, $\mathrm{N}(\%)$ & & & * \\
\hline Spring & $730(27.7 \%)$ & $3,944(29.9 \%)$ & \\
\hline Summer & $401(15.2 \%)$ & $2,332(17.7 \%)$ & \\
\hline Fall & $211(8.0 \%)$ & $1,605(12.2 \%)$ & \\
\hline Winter & $1,291(49.0 \%)$ & $5,292(40.2 \%)$ & \\
\hline \multicolumn{4}{|l|}{ Primary care access, N (\%) } \\
\hline Coordination of primary care & $326(12.4 \%)$ & $1,747(13.3 \%)$ & \\
\hline \multicolumn{4}{|l|}{ Continuity of primary care, N (\%) } \\
\hline Complete care continuity & $349(13.3 \%)$ & $1,764(13.4 \%)$ & \\
\hline Some care continuity & $634(24.1 \%)$ & $2,960(22.5 \%)$ & \\
\hline No care continuity & $1650(62.7 \%)$ & $8,449(64.1 \%)$ & \\
\hline \multicolumn{4}{|l|}{ Healthcare utilization, N (\%) } \\
\hline Emergency room visit & $893(33.9 \%)$ & $4,449(33.8 \%)$ & \\
\hline \multicolumn{4}{|l|}{ County-level characteristics } \\
\hline \multicolumn{4}{|l|}{ Metropolitan status, N (\%) } \\
\hline Nonmetro & $267(10.1 \%)$ & $1,285(9.8 \%)$ & \\
\hline Metro & $2,366(89.9 \%)$ & $11,888(90.2 \%)$ & \\
\hline Primary care shortage area, N (\%) & & & $\dagger$ \\
\hline Whole county & $2,034(77.3 \%)$ & $10,147(77.0 \%)$ & \\
\hline Part county & $429(16.3 \%)$ & $2,312(17.6 \%)$ & \\
\hline No shortage & $170(6.5 \%)$ & $714(5.4 \%)$ & \\
\hline Mental healthcare shortage area, $\mathrm{N}(\%)$ & & & $\ddagger$ \\
\hline Whole county & $2,015(76.5 \%)$ & $9,925(75.3 \%)$ & \\
\hline Part county & $388(14.7 \%)$ & $2,242(17.0 \%)$ & \\
\hline No shortage & $230(8.7 \%)$ & $1,006(7.6 \%)$ & \\
\hline CMHC, mean [SD] & $0.81[1.23]$ & $0.94[1.24]$ & * \\
\hline Rural health center, mean [SD] & $0.62[3.03]$ & $1.06[4.41]$ & * \\
\hline FQHC, mean [SD] & $37.69[44.31]$ & $37.78[42.98]$ & \\
\hline Education rate, $4+$ years, mean [SD] & $25.39[10.98]$ & $23.77[10.51]$ & * \\
\hline Unemployment rate, mean [SD] & $4.57[0.71]$ & $4.67[0.90]$ & * \\
\hline \% Below poverty level, mean [SD] & $15.11[3.73]$ & $15.06[3.80]$ & \\
\hline Per capita income (US dollars), mean [SD] & $58,761.96[33,697.42]$ & $54,029.16[31,265.86]$ & * \\
\hline Nonfederal PCP density, mean [SD] & $307.10[192.29]$ & $279.97[179.22]$ & * \\
\hline Hospital density, mean [SD] & $1.74[1.37]$ & $1.65[1.14]$ & * \\
\hline
\end{tabular}

NOTE: Based on 15,806 nonelderly (21-64 years old) fee-for-service Medicaid beneficiaries residing in California, Illinois, New York, and Texas with selected chronic conditions, who were alive and had continuous fee-for-service enrollment through the observation period, were not enrolled in Medicare, and had at least 1 inpatient encounter in the follow-up period. Significant group differences in all-cause 30 -day readmissions were tested with $\chi^{2}$ and $t$ tests. Asterisks represent significant group differences between the 30-day readmission and no 30-day readmission groups. Column percentages are reported for categorical variables. Abbreviations: Al, American Indian; $\mathrm{CMHC}$, community mental health center; COPD, chronic obstructive pulmonary disease; FQHC, federally qualified health center; HIV, human immunodeficiency virus; PCP, primary care practitioners; PI, Pacific Islander; SD, standard deviation; US, United States. ${ }^{*} P<0.001 .+0.01 \leq P<0.05$. $t 0.001 \leq P<0.01$. 


\begin{tabular}{|c|c|c|c|}
\hline & AUR & $95 \%$ & Signiticance \\
\hline Length of stay & 1.03 & [1.03-1.04] & * \\
\hline Arthritis/osteoporosis & 0.90 & {$[0.72-1.13]$} & \\
\hline Cancer & 1.55 & {$[1.26-1.90]$} & * \\
\hline Cardiovascular conditions & 1.20 & {$[1.08-1.33]$} & * \\
\hline COPD/asthma & 1.01 & {$[0.90-1.12]$} & \\
\hline Diabetes & 1.23 & {$[1.10-1.39]$} & * \\
\hline HIV/hepatitis & 0.98 & {$[0.85-1.12]$} & \\
\hline Mental health conditions & 2.17 & {$[1.98-2.38]$} & * \\
\hline \multicolumn{4}{|l|}{ Season of readmission } \\
\hline Spring & 0.79 & {$[0.71-0.88]$} & * \\
\hline Summer & 0.77 & {$[0.68-0.88]$} & * \\
\hline Fall & 0.58 & {$[0.49-0.68]$} & * \\
\hline Winter & Reference & & \\
\hline Cash eligibility & 1.14 & {$[1.01-1.27]$} & $\dagger$ \\
\hline Medical need eligibility & 1.21 & {$[1.08-1.36]$} & $\dagger$ \\
\hline
\end{tabular}

NOTE: Based on 15,806 nonelderly (21-64 years old) fee-for-service Medicaid beneficiaries residing in California, Illinois, New York, and Texas with selected chronic conditions, who were alive and had continuous fee-for-service enrollment through the observation period, were not enrolled in Medicare, and had at least 1 inpatient encounter in the follow-up period. Model controlled for patient-level, and county-level variables. Patient-level variables were demographic (gender, age, race/ethnicity), Medicaid eligibility characteristics (cash and medical need), primary care access (continuity and coordination of care), and healthcare utilization (emergency room visits). County-level variables were metropolitan statistical area, primary care shortage areas, mental healthcare shortage areas, community mental health centers, rural health centers, federally qualified health centers, college education rate, unemployment rate, poverty level, per capita income, density of primary care providers, and hospital density. Asterisks represent significant group differences in 30-day readmission compared to the reference group. The logistic regressions also included intercept terms. The regressions accounted for clustering of individuals within counties. Abbreviations: AOR, adjusted odds ratio; $\mathrm{Cl}$, confidence interval; $\mathrm{COPD}$, chronic pulmonary obstructive disease; HIV, human immunodeficiency virus. ${ }^{*} P<0.0001 . \dagger 0.01 \leq P<0.05 . \$ 0.001 \leq P<0.01$.

such as accountable care organizations and patientcentered medical homes, which offer comprehensive, well-coordinated primary care services, may be needed to reduce readmission among Medicaid beneficiaries with chronic health conditions. In this respect, 3 of the 4 states represented (California, New York, and Texas) are CMS Innovation Model partner states and are presently awardees of Medicaid Incentives for the Prevention of Chronic Disease state grants. ${ }^{20}$ It remains to be seen whether such programs can reduce the high prevalence of readmissions in a Medicaid population.

Although our findings may have implications in reducing readmission risk, these results need to be interpreted in the light of study limitations. Our study was based on beneficiaries from only 4 states and cannot be generalized to the entire US Medicaid population. We also excluded individuals who were not enrolled in Medicaid health maintenance organizations. Given that less than one-third of the population receives fee-for-service care in Medicaid, our study may have selection bias. Our study design utilized a retrospective cohort approach and cannot be used to establish causal relationships. Further, our study did not include adjustment for variables related to discharge planning or care coordination other than a primary care visit 14 days post discharge, which might influence the readmission risk of complex patients. Our study utilized data from administrative claims files.

Overall, our analyses revealed that patient complexities increased the risk of all-cause 30-day readmission for high-risk Medicaid beneficiaries with chronic conditions, thus warranting the need for comprehensive care for those with chronic conditions. Programs designed to reduce the risk of 30-day readmissions may need to focus on appropriate disease management and better coordinated care post hospitalization.

Disclosures: Research reported in this publication was supported by the Training Program in the Behavioral and Biomedical Sciences at West Virginia University, National Institute of General Medical Sciences grant number T32 GM08174, and the National Institute of General Medical Sciences of the National Institutes of Health under award number U54GM104942, and the Benedum Foundation. The content is solely the responsibility of the author and does not necessarily represent the official views of the National Institutes of Health. The funders had no role in study design, data collection and analyses, decision to publish, or preparation of the manuscript.

\section{References}

1. Podulka J, Barrett M, Jiang HJ, et al. 30-day readmissions following hospitalizations for chronic vs. acute conditions, 2008: statistical brief \#127. In: Healthcare Cost and Utilization Project Statistical Briefs. Rockville, MD: Agency for Health Care Policy and Research. Available at: http://www.ncbi.nlm.nih.gov/books/NBK92612. Published February 2012. Accessed August 20, 2015.

2. Axon RN, Williams MV. Hospital readmission as an accountability measure. JAMA. 2011;305:504-505.

3. Hines AL, Barrett ML, Jiang HJ, et al. Conditions with the largest number of adult hospital readmissions by payer, 2011: statistical brief \#172. In: Healthcare Cost and Utilization Project Statistical Briefs. Rockville, MD: Agency for Health Care Policy and Research. Available at: http://www.ncbi.nlm.nih.gov/books/NBK206781. Published April 2014. Accessed September 15, 2015.

4. Mark TL, Tomic KS, Kowlessar N, et al. Hospital readmission among medicaid patients with an index hospitalization for mental and/or substance use disorder. J Behav Health Serv Res. 2013;40:207-221.

5. Trudnak T, Kelley D, Zerzan J, et al. Medicaid admissions and readmissions: understanding the prevalence, payment, and most common diagnoses. Health Aff (Millwood). 2014;33:1337-1344.

6. Allen SM, Croke AL. The Faces of Medicaid: The Complexities of Caring for People With Chronic Illness and Disabilities. Available at: http://www.chcs.org/usr_doc/Chartbook.pdf. Published October 2000. Accessed November 17, 2014.

7. Wier LM, Barrett M, Steiner C, et al. All-cause readmissions by payer and age, 2008: statistical brief \#115. In: Healthcare Cost and Utilization Project Statistical Briefs. Rockville, MD: Agency for Health Care Policy and Research. Available at: http://www.ncbi.nlm.nih.gov/ books/NBK56036. Published June 2011. Accessed September 15, 2015.

8. Eapen ZJ, Reed SD, Li Y, et al. Do countries or hospitals with longer hospital stays for acute heart failure have lower readmission rates?: Findings from ASCEND-HF. Circ Heart Fail. 2013;6:727-732.

9. Carey K, Lin MY. Hospital length of stay and readmission: an early investigation. Med Care Res Rev. 2014;71:99-111.

10. Kaboli PJ, Go JT, Hockenberry J, et al. Associations between reduced hospital length of stay and 30-day readmission rate and mortality: 14year experience in 129 Veterans Affairs hospitals. Ann Intern Med. 2012;157:837-845.

11. Garrison GM, Mansukhani MP, Bohn B. Predictors of thirty-day readmission among hospitalized family medicine patients. J Am Board Fam Med. 2013;26:71-77.

12. Khan S, Kalogeropoulos AP, Ambrosy, AP. Hospital length of stay and readmissions post heart failure hospitalization in the EVEREST Trial. Circulation. 2014;130: A12794. Available at: http://circ.ahajournals.org/content/130/Suppl_2/A12794. Accessed September 15, 2015

13. Garcia-Perez L, Linertova R, Lorenzo-Riera A, et al. Risk factors for hospital readmissions in elderly patients: a systematic review. OJM. 2011;104:639-651. 
14. Jencks SF, Williams MV, Coleman EA. Rehospitalizations among patients in the Medicare fee-for-service program. N Engl J Med. 2009; 360:1418-1428.

15. Andersen RM. National health surveys and the behavioral model of health services use. Med Care. 2008;46:647-653.

16. Goodman RA, Posner SF, Huang ES, et al. Defining and measuring chronic conditions: imperatives for research, policy, program, and practice. Prev Chronic Dis. 2013;10:E66.

17. McMartin K. Discharge planning in chronic conditions: an evidencebased analysis. Ont Health Technol Assess Ser. 2013;13:1-72.
18. Kasper EK, Gerstenblith G, Hefter G, et al. A randomized trial of the efficacy of multidisciplinary care in heart failure outpatients at high risk of hospital readmission. J Am Coll Cardiol. 2002;39:471-480.

19. Casas A, Troosters T, Garcia-Aymerich J, et al. Integrated care prevents hospitalisations for exacerbations in COPD patients. Eur Respir J. 2006;28:123-130.

20. Centers for Medicare \& Medicaid Services. Readmissions Reduction Program. Available at: http://www.cms.gov/Medicare/Medicare-Feefor-Service-Payment/AcuteInpatientPPS/Readmissions-Reduction-Program.html. Accessed February 27, 2015. 